\title{
Fine-tuning the Cross-Sectional Architecture of Antimony- doped Tin Oxide Nanofibers as Pt Catalyst Support for Enhanced Oxygen Reduction Activity
}

\author{
Shuxiao Chen ${ }^{1,2}$, Hongda $\mathrm{Du}^{1,2}$, Yinping Wei ${ }^{1}$,Lingyi Peng ${ }^{1}$, Yadong $\mathrm{Li}^{1}$, Lin Gan ${ }^{1, *}$, \\ Feiyu Kang ${ }^{1,2, *}$ \\ ${ }^{1}$ Division of Energy and Environment, Graduate School at Shenzhen, Tsinghua University, Shenzhen, \\ 518055, PR China \\ ${ }^{2}$ Guangdong Provincial Key Laboratory of Thermal Management Engineering \& Materials, Graduate \\ School at Shenzhen, Tsinghua University, Shenzhen, 518055, PR China \\ *E-mail: 1gan@sz.tsinghua.edu.cn, fykang@sz.tsinghua.edu.cn
}

doi: $10.20964 / 2017.07 .42$

Received: 31 March 2017 / Accepted: 11 May 2017 / Published: 12 June 2017

We report the fabrication of electrospun antimony-doped tin oxides (ATO) nanofibers (NFs) with controlled cross-sectional architectures ranging from solid dense NFs via fiber-in-tube NFs to completely hollow tube-like NFs at otherwise constant conditions. These ATO NFs were further applied as catalyst supports on which Pt nanoparticles were electrodeposited. Our results show that the Pt nanoparticles deposited on the hollow ATO NFs exhibited a higher catalytic activity on oxygen reduction reaction compared to those supported on solid ATO NFs and conventional ATO powders. Transmission electron microscopy revealed that the Pt nanoparticles deposited on the hollow ATO NFs were spontaneously confined into the hollow channels of the NFs, which likely induced a nanoconfinement effect and hence higher catalytic activity. Our results provide important insight into rational control of the mesostructures of metal oxides supported fuel cell catalysts for enhance catalytic properties.

Keywords: electrospun nanofibers; antimony-doped tin oxides ; Pt nanoparticles; oxygen reduction reaction; nano-confinement effect

\section{$\underline{\text { FULL TEXT }}$}

(C) 2017 The Authors. Published by ESG (www.electrochemsci.org). This article is an open access article distributed under the terms and conditions of the Creative Commons Attribution license (http://creativecommons.org/licenses/by/4.0/). 